\title{
Métricas para análise de poder em redes sociais e sua aplicação nas doações de campanha para o Senado Federal brasileiro
}

\author{
Letícia Verona $^{1}$, Jonice Oliveira ${ }^{1}$, Maria Luiza Machado Campos $^{1}$ \\ Programa de Pós-Graduação em Informática (PPGI) \\ Universidade Federal do Rio de Janeiro - (UFRJ) - Rio de Janeiro - RJ \\ leticiaverona@ufrj.br, jonice@dcc.ufrj.br, mluiza@nce.ufrj.br
}

\begin{abstract}
The analysis of how much one actor has over another is crucial to understanding what interests their actions are subordinate to. This paper presents metrics based on the theoretical bases of sociology that reflect the imbalance between relationships, the main source of power in a social network. These metrics are applyed to the relationship between each pair of nodes, and are computed to accomplish the entire power structure of the network. As an instrument of empirical validation, a case study was made with the electoral donations received by parties and senators in the campaigns of 2010 and 2014. The application of the metrics allowed to highlight some corporate and party interests existing today in the Brazilian Federal Senate.
\end{abstract}

Resumo. A análise de quanto poder um ator tem sobre o outro é fundamental para entender a que interesses suas ações estão subordinadas. Este trabalho apresenta métricas para a análise fundamentado em bases teóricas da sociologia que refletem o desequilíbrio entre as relações, principal fonte de poder em uma rede social. Métricas que se aplicam ao relacionamento entre cada par de nós, e são calculadas em relação a toda a estrutura de poder da rede. Como instrumento de validação empírico foi feito estudo de caso com as doações eleitorais recebidas por partidos e senadores nas campanhas de 2010 e 2014. A aplicação das métricas permitiu evidenciar alguns interesses corporativos e partidários existentes hoje no Senado Federal brasileiro.

\section{Introdução}

Poder é a capacidade de um agente de impor a sua vontade sobre a de outro, baseando-se em forças de dominação intrínsecas à sociedade [Castells 2011]. Um dos aspectos evidenciado por teorias sociológicas, como em [Emerson 1976] é que o poder em uma rede social é um processo dinâmico, um embate entre forças que competem para obter mais influência em seu favor. $\mathrm{O}$ controle e a troca de recursos são pontos pivotais na obtenção de poder. Sejam recursos financeiros, controle de disseminação de mídia, regulamentação de leis ou qualquer outro recurso de valor para a rede, quem controla e negocia estes recursos se fortalece. Para realizar essas trocas são necessárias habilidades específicas para a sociedade em rede: fazer conexões com outras redes, proteger seus parceiros de outros competidores, e se possível, controlar os protocolos de negociação e comunicação. Buscamos através destas bases teóricas analisar o poder estabelecido em uma rede onde a 
transferência de recursos ocorre: a rede formada pelas doações de campanha ao Senado em 2010 e 2014.

A natureza competitiva da obtenção de poder é relacional, logo o poder não é um atributo absoluto de um ator da rede. O poder é exercido por um agente sobre outro e deve ser medido em relação a aresta, e não aos nós da rede. Para medir este poder em uma rede de troca de recursos propomos explorar uma métrica de avaliação de quanto a troca em questão é importante para cada um dos nós da relação. Para tal, considerando que a rede é direcional, criamos duas medidas auxiliares: influencia política (political influence) e barganha (bargain), ambas calculadas sobre a aresta, em direções opostas. $\mathrm{O}$ poder (power) é a relação (ou desequilíbrio) entre estes dois fatores. Para ser aderente às bases teóricas utilizadas, uma premissa é que a aresta tenha o significado específico, que é a transferência de recursos de valor entre os nós.

A rede social das doações de campanha para o Senado Federal brasileiro nas campanhas de 2010 e 2014 foi analisada, buscando entender quais são os interesses e forças que atuam sobre os legisladores e o quanto sua independência pode ser aferida. Os resultados da análise mostraram que as grandes empresas doadoras concentram suas doações em grandes partidos, buscando controle global e diversificado sobre os senadores. Empresas locais e de menor porte fazem doações mais personalizadas a comitês individuais. Alguns senadores possuem uma situação de independência e outros têm um vínculo mais estreito com o partido. Por fim, partidos grandes atuam como pontes de recursos entre as grandes empresas e os partidos menores, obtendo poder sobre eles. $\mathrm{O}$ estudo permitiu a obtenção de um mapa de intenções e interesses do Senado e mostrou ser eficiente para entender as dinâmicas de troca de recursos.

O restante do artigo está organizado da seguinte maneira: na Seção 2 é apresentada a formulação teórica sobre poder. Na Seção 3 o processo de análise é detalhado e suas fórmulas apresentadas. O estudo de caso e a rede social do Senado Federal são apresentados na Seção 4. Na Seção 5 são apresentados os resultados da análise e finalmente, na Seção 6 são discutidas as limitações do trabalho e sugeridas futuras análises.

\section{Abordagens para análise de poder}

Poder é um conceito amplamente discutido em diversas áreas das ciências humanas, como a filosofia e a psicologia. Este estudo possui foco na avaliação de poder em redes sociais e para embasar a análise realizada buscamos trabalhos cujo aspecto social do poder fosse discutido. As causas e feitos do poder em relações sociais estão presentes em trabalhos das áreas de sociologia e economia há décadas [Marx and Engels 1968] [Hindess 1996] [French et al. 1959]. O tema foi re-potencializado pela configuração da sociedade em rede nas últimas décadas e passou a interessar pesquisadores da Ciência da Computação. Está fora do escopo deste estudo uma revisão de literatura sobre um assunto tão vasto, porém algumas correntes principais de pensamento são descritas a seguir, especialmente aquelas em cujas bases o presente estudo se apoia.

Manuel Castells talvez seja o sociólogo mais influente na análise e compreensão da sociedade contemporânea como uma sociedade em rede. Apesar disto, curiosamente a interface do seu trabalho com a área de análise de redes sociais é pequena, como detalhado em [Anttiroiko 2015]. Para ele, a rede baseada nas tecnologias de informação possibilitou organizacionalmente a transformação da sociedade. Sociedade que migrou das 
relações fundamentadas em localização espacial e em relações de produção para fluxos de informação e poder. Um de seus trabalhos [Castells 1996b] é um marco na representação abstrata da sociedade como uma rede de nós interconectados. Enfatiza que esta sociedade conectada é uma sociedade capitalista em progressão, onde o capital se torna mais forte ao formar redes, enquanto o lado trabalho se torna mais fraco com o individualismo crescente [Castells 1996a]. Vai além, dizendo que os processos globais são cada vez mais regidos por redes econômicas e de informação que medem o valor de pessoas, grupos sociais e até países por sua utilidade (use value) na rede.

Ainda para Castells, o poder segue a lógica da construção da rede. "Em um mundo conectado, a habilidade de exercitar controle sobre os outros reside em dois mecanismos básicos: (a) a habilidade de programar ou reprogramar as redes em termos de seus objetivos e protocolos; e (b) a habilidade de conectar e assegurar a cooperação entre diferentes redes através da combinação de recursos ao mesmo tempo que isola de outras através de competição estratégica" [Castells 2011]. Estas duas habilidades estão nomeadas como programming e switching respectivamente. Ao regular as redes sociais online através da constante reformulação de protocolos ou formatos de mensagens, corporações de mídia estão exercendo seu poder de programação, pois as mensagens devem se adaptar ao novo formato imposto (técnico ou de conteúdo). Assim como [MacLuhan et al. 1967], Castells coloca o poder da comunicação no meio e não no conteúdo. Estas corporações decidem sobre o conteúdo e formato a partir do que vai ao encontro de seus interesses e dos objetivos que eles determinam para a rede: lucro, poder e fabricação de cultura.

Para [Emerson 1976] o poder é definido como uma capacidade estrutural que promove a distribuição desigual de recursos, favorecendo alguns atores em detrimento de outros. Em sua Teoria do Poder-Dependência ele enfatiza que o poder é uma propriedade relacional dependente dos recursos trocados e controlados. Atores que têm múltiplas possibilidades de parceiros para negociação e controlam recursos de alto valor são tidos como poderosos na sua rede de troca. O poder dos indivíduos é interdependente, pois está implícito nas relações sociais.

Em [Willer 1999] estes elementos - controle de recursos e opções de negociação são enriquecidos com o elemento da vantagem, supondo que atores que possuem opções alternativas vantajosas na negociação obtém mais poder no relacionamento através de mecanismos de confrontação. Em [Easley and Kleinberg 2010] os autores discutem como o poder se manifesta entre pares ligados por arestas em uma rede social através de uma relação de troca. Mais ainda, em como indica a ascendência de um nó sobre o outro, especialmente o desequilíbrio da relação.

Especificamente, agentes políticos possuem controle sobre regulamentações, empréstimos de bancos públicos, gestão de obras e muitos outros recursos de grande valor. Para obterem suas posições, através de nomeações ou eleições precisam de recursos financeiros obtidos de outros agentes (por exemplo organizações religiosas, comerciais, militares) cujos interesses ele representam. Nesta relação, o político busca maximizar sua autonomia e o agente externo busca aumentar sua chance de manipulação. [Castells 1996b].

Trabalhos recentes usaram dados de doações de campanha a políticos para analisar a conexão de políticos com corporações. Em [Mancuso et al. 2016] os dados de doações 
nas eleições para deputado federal em 2010 são usados para indicar quais são os partidos mais dependentes das corporações em geral dentro do espectro político brasileiro. No estudo [Boas et al. 2014] é explicitada a relação entre as doações de campanha e contratos firmados com o poder público. Também é destacada a longeva relação entre políticos de carreira e determinadas empresas, mostrando uma relação de benefício mútuo, frequentemente desvinculada dos interesses da população. O presente estudo difere dos citados pois se propõe calcular o grau de dependência/independência que cada partido ou senador possui.

\section{Métricas para a aresta: influência, barganha e poder}

Com estes conceitos teóricos no horizonte propomos a criação da métrica poder (power), com três características importantes: (i) as hipóteses sobre a natureza do poder herdam conceitos sociológicos e assumem que o significado da aresta é a transferência de recursos dentro da rede; (ii) é uma métrica relacional e portanto atribuída às arestas e não aos nós; e (iii) é relativa e não absoluta, de maneira que a mudança em uma aresta se propaga e afeta todas as relações de poder da rede. Foram criadas duas métricas auxiliares que atuam sobre a relação entre os nós: influência política (political influence) e poder de barganha (bargain). Estas métricas possuem a mesma mecânica e a distinção de nomenclatura reflete apenas a direção da aresta, que reflete de onde vem e para onde vai o recurso financeiro.

A influência política (political influence) do nó $a$ em relação ao nó $b$ é um atributo da aresta na mesma direção da mesma. Na Equação 1, Wedge $e_{a b}$ é o peso da aresta que liga $a$ e $b$, e Windegree $_{b}$ é o grau ponderado de entrada, que corresponde ao total de recursos recebido por $b$. Significa o quanto esta troca com $a$ representa em relação a tudo que $b$ recebeu. Quanto maior este número, mais $a$ influencia as decisões de $b$.

Simetricamente, o poder de barganha (bargain) aplica uma relação no sentido oposto da aresta, ou seja, no sentido oposto da transferência de recursos, como pode ser visto na Equação 2. Indica o quanto esta mesma troca com $b$ representa sobre o total de recursos transferidos por $a$.

$$
\begin{gathered}
\text { politicalInfluence }_{a b}=\left(\text { Wedge }_{a b} / \text { Windegree }_{b}\right) \\
\text { bargain }_{b a}=\left(\text { Wedge }_{a b} / \text { Woutdegree }_{a}\right)
\end{gathered}
$$

Finalmente, o poder relacionado com a aresta segue na mesma direção da mesma e deve refletir o desequilíbrio entre estes dois fatores. Quanto menos equilibrada a relação, mais empoderado será um dos nós no relacionamento. O poder (power - $\Psi_{a b}$ ) de $a$ sobre $b$ pode ser descrito pela Equação 3.

$$
\Psi_{a b}=\text { politicalInfluence }_{a b}-\text { bargain }_{b a}
$$

A relação é mais equilibrada quando a $\Psi_{a b}$ se aproxima de 0 . Valores acima de 0 indicam que o nó que é a fonte de recursos é mais poderoso e valores abaixo de 0 indicam que apesar de estar recebendo recursos, o nó destino tem mais poder. Como exemplo de 
cálculo seguindo este método, podemos examinar uma rede de troca de recursos simples como a exibida na Figura 1. Queremos responder sobre quais interesses se dá a atuação do nó D. Para fazer isto medimos a relação deste nó com os vizinhos que transferem recursos para ele, como pode ser visto nos cálculos a seguir.

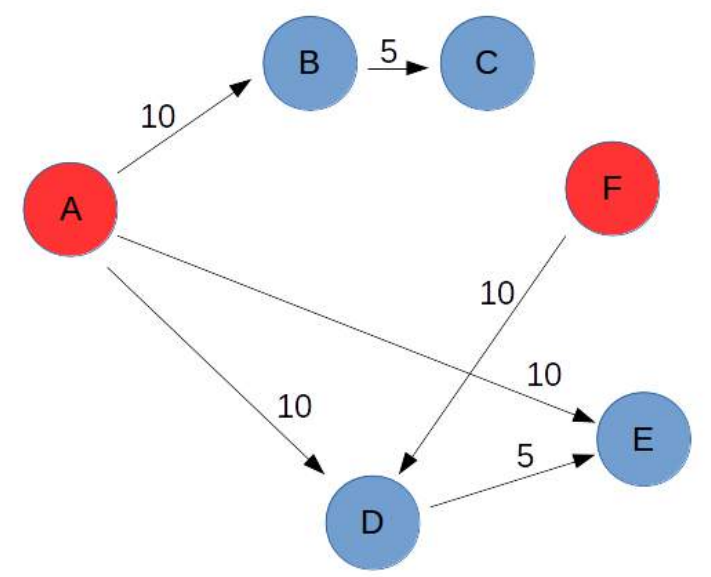

Figura 1. Exemplo de uma rede de troca de recursos simples

$$
\begin{gathered}
\text { politicalInfluence }_{a d}=10 / 20=0,5 \\
\text { politicalInfluence }_{f d}=10 / 20=0,5 \\
\text { bargain }_{d a}=10 / 30=0,33 \\
\text { bargain }_{d f}=10 / 10=1
\end{gathered}
$$

Como o peso das arestas vindas de A e F são iguais, a influência política destes nós sobre D é idêntica. Porém como A possui outras opções de troca, o poder de barganha de $\mathrm{D}$ com este nó é menor do que com $\mathrm{F}$, que só troca com ele e isto interfere decisivamente no poder exercido sobre D.

$$
\begin{gathered}
\Psi_{a d}=0,5-0,33=0,17 \\
\Psi_{f d}=0,5-1=-0,5
\end{gathered}
$$

\section{A rede social do Senado Federal e seus doadores eleitorais}

A Lei de Acesso à Informação, Lei $n^{\circ} 12.527$, de 18.12.2011 garante ao cidadão brasileiro o acesso a qualquer informação pública que esteja dentro dos limites descritos na lei. Candidatos a cargos executivos e legislativos devem fornecer à justiça eleitoral a prestação de contas de sua campanha e o Superior Tribunal Eleitoral através do seu Repositório de Dados Eleitorais disponibiliza esta informação publicamente [Tribunal Superior Eleitoral 2016]. Esses dados na sua forma bruta são difíceis de entender, analisar e visualizar. Este estudo busca trazer à tona as relações de poder que envolvem os senadores e as empresas mostrando o que motiva decisões que afetam toda 
a população. Para tal, as doações recebidas pelos atuais senadores e seus partidos são o objeto desta análise. O objetivo é determinar o poder que as doações de cada empresa geram.

A rede social dos senadores, partidos e doadores construída sobre estes dados e baseada no valor das doações busca responder às seguintes questões: (i) quais são as empresas que mais doam e como distribuem suas doações; (ii) qual é o papel dos partidos e das suas lideranças na distribuição das doações; (iii) qual o grau de poder que cada empresa possui sobre cada senador.

Para discutirmos como as métricas se aplicam neste caso: uma empresa tem influência política sobre o partido ou o senador que recebe os seus recursos. Um partido pode ter influência política sobre o outro, através da transferência de doações. A influência política, conforme discutido na Seção 3, é calculada usando a relação entre o peso da aresta em questão e o total de doações recebidas por este nó, considerando que os nós competem entre si. Simetricamente, se a empresa doa para somente um senador, o mesmo ganha poder de barganha com a empresa em questão.

Um senador pode ter muito poder de barganha sobre uma empresa, pois a mesma concentra suas doações nele. Entretanto se o senador depende dessas doações de maneira significativa, temos um equilíbrio na relação e portanto o nível de poder da empresa sobre o senador é pequeno. Um senador que depende exclusivamente do seu partido está em uma situação de poder em relação ao partido muito diferente daquele que financia sua própria campanha. Um senador que tem muitos grandes financiadores tem uma posição mais empoderada em relação a cada empresa do que aquele que depende exclusivamente de uma empresa para viabilizar seus gastos de campanha.

\subsection{Metodologia de construção da rede social}

Para construção da rede social dos senadores, partidos e seus doadores foram utilizados os dados do Tribunal Superior Eleitoral com as doações de campanha para as eleições de 2010 e 2014. Para efeito desta análise foram usadas as doações aos partidos e comitês eleitorais e as doações diretas aos comitês de campanha dos senadores. Os dados utilizados foram limitados a doações acima de dez mil reais. Cabe ressaltar que não foram incluídas na rede doações feitas para comitês individuais de cargos do executivo e de candidatos a deputado estadual e federal.

Com estes dados foram criados nós para cada senador, cada partido e cada doador. Ligando estes nós, arestas direcionadas na direção da doação e com o peso correspondente ao valor doado. Em alguns registros dos dados era indicado o doador originário. Neste caso a doação foi transformada em duas arestas, uma para a doação original (da empresa para o comitê original) e outra para a transferência (do comitê original para o segundo comitê). Partidos políticos possuem vários CNPJs espalhados por diretórios regionais que foram unificados no nó que corresponde ao partido.

Para realização deste estudo foi utilizado o software Gephi com o plugin SigmaJS para geração da visualização da rede.

\subsection{Resultados}

A rede construída pode ser visualizada na Figura 2. Para efeitos de visualização, a cor do nó indica o tipo de nó (partido, senador ou doador) e o tamanho do nó é calculado 
com base no grau ponderado do mesmo, que neste caso significa também o montante em dinheiro que trafegou por aquele nó.

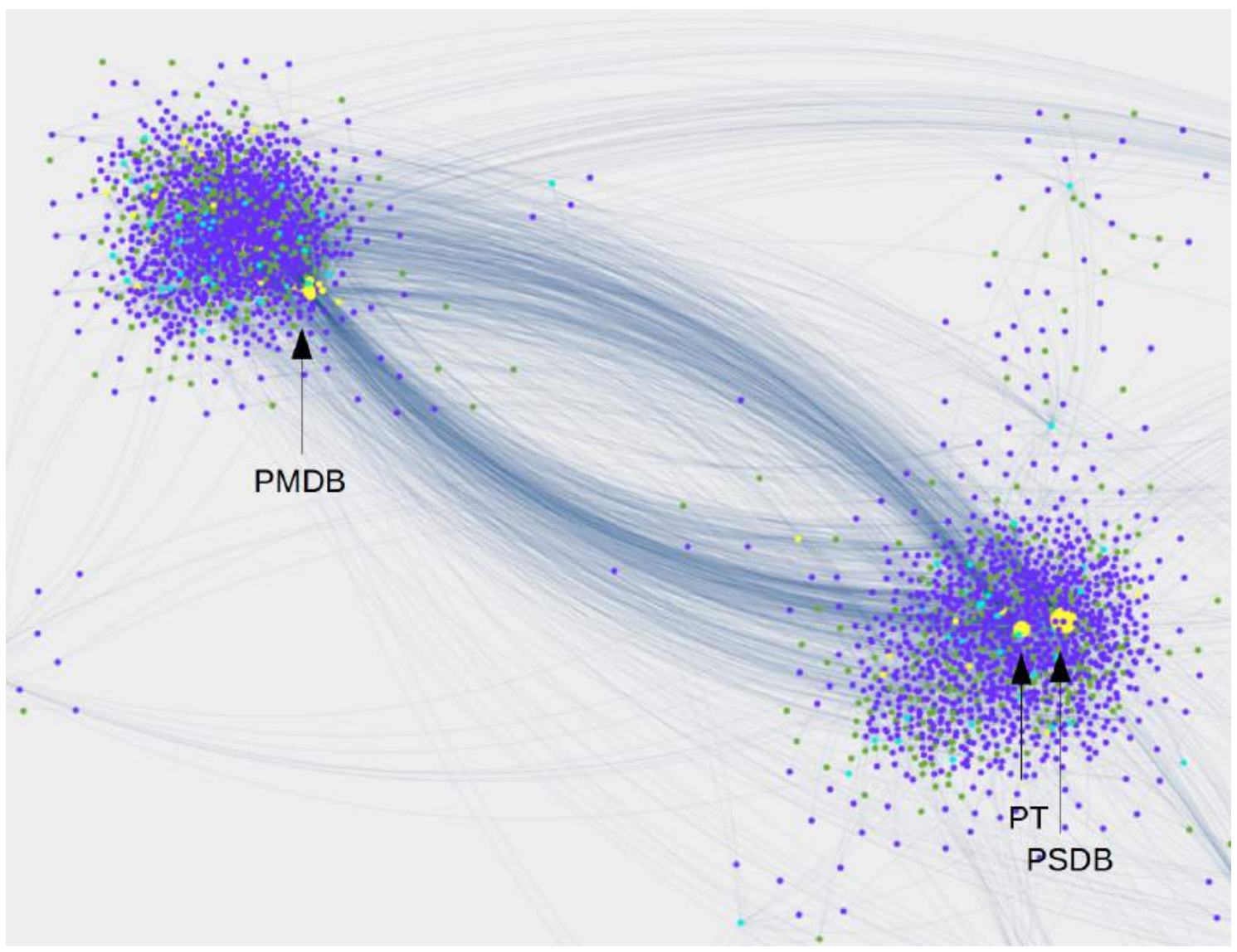

Figura 2. Detalhe da rede social do Senado Federal: partidos e doadores de campanha, indicando a posição dos três maiores partidos.

A visualização da rede gerada está disponível ${ }^{1}$, bem como os dados que geram o grafo representando a rede gerada em formato gephi ${ }^{2}$.

\section{Análise de Resultados}

Antes de aplicar as métricas de poder, analisamos a rede do Senado em relação a algumas métricas tradicionais de análise de redes sociais. Primeiro, foram verificadas as maiores empresas doadoras. A presença de duas empresas de alimentos na lista das dez maiores empresas doadoras chamou a atenção, além do já esperado maciço volume de dinheiro vindo de empreiteiras.

Em relação às métricas, notamos que em alguns casos o volume de dinheiro doado (grau ponderado de saída) não tem uma relação direta com a métrica de $h u b$ do nó. Um exemplo é mostrado na Tabela 1: a Construtora Andrade Gutierrez S.A., apesar de ter efetuado quase o dobro de doações em volume de dinheiro possui um índice $h u b$ menor que a Construtora Norberto Odebrecht S.A..

\footnotetext{
${ }^{1}$ http://bit.ly/2pDFPtd

${ }^{2}$ http://bit.ly/2ppIk6H
} 
Tabela 1. Maiores empresas doadoras

\begin{tabular}{llrrr}
\hline Empresa & Ramo & Grau saída & Grau ponderado (saída) & Hub \\
\hline JBS S.A. & Alimentos e Bebidas & 39 & $\mathrm{R} \$ 431.835 .228,00$ & 0,098 \\
Construtora Andrade Gutierrez S.A. & Empreiteira & 15 & $\mathrm{R} \$ 212.460 .000,00$ & 0,081 \\
Construtora Queiroz Galvão S.A. & Empreiteira & 16 & $\mathrm{R} \$ 126.908 .162,00$ & 0,087 \\
Construtora OAS S.A. & Empreiteira & 23 & $\mathrm{R} \$ 105.303 .088,00$ & 0,082 \\
Construtora Norberto Odebrecht S.A. & Empreiteira & 25 & $\mathrm{R} \$ 95.937 .824,00$ & 0,092 \\
Arcelormittal Brasil S.A. & Minério e aço & 20 & $\mathrm{R} \$ 81.015 .280,00$ & 0,079 \\
UTC Engenharia S.A. & Empreiteira & 21 & $\mathrm{R} \$ 61.478 .640,00$ & 0,079 \\
Carioca Nielsen Engenharia S.A. & Empreiteira & 19 & $\mathrm{R} \$ 59.680 .000,00$ & 0,086 \\
Cervejaria Petrópolis S.A. & Alimentos e Bebidas & 9 & $\mathrm{R} \$ 54.669 .608,00$ & 0,047 \\
Amil Assistência Médica Internacional S.A. & Saúde & 11 & $\mathrm{R} \$ 53.148 .000,00$ & 0,075 \\
\hline
\end{tabular}

As grandes empresas concentram suas doações em partidos e não em comitês individuais. Uma interpretação seria a grande força dos líderes de partidos junto a estas empresas. Outra interpretação possível é que as empresas buscam dissimular seu vínculo direto com um ou outro senador. Na Tabela 2 podem ser vistos os montantes recebidos pelos três partidos mais fortes do Congresso Nacional.

\begin{tabular}{|c|c|c|c|c|}
\hline Partido & Grau Entrada & Grau ponderado Entrada & Authority & Hub \\
\hline PSDB & 746 & $\mathrm{R} \$ 861.763 .078,00$ & 0,601 & 0,057 \\
\hline PMDB & 579 & $\mathrm{R} \$ 857.877 .510,00$ & 0,444 & 0,070 \\
\hline PT & 472 & $\mathrm{R} \$ 397.102 .206,00$ & 0,396 & 0,034 \\
\hline
\end{tabular}

Outro item curioso que a rede nos mostra é que os grandes partidos (PMDB, PSDB e PT) atuam como financiadores de campanha dos partidos menores, atuando como intermediários entre os mesmos e as grandes empresas. Claramente podemos ver aqui um esforço para obter o que Castells chamou de poder de switching, onde o nó que faz a conexão entre redes ganha força e protege seus interesses. Na Tabela 3 podemos ver que entre os 10 maiores destinos dos grandes partidos estão outros partidos.

Tabela 3. Maiores destinos de recursos dos partidos PMDB, PSDB e PT

\begin{tabular}{lrlrlr}
\hline PMDB & Valor doado & PSDB & Valor doado & PT & Valor doado \\
\hline PSB & $\mathrm{R} \$ 18.075 .976,00$ & José Serra & $\mathrm{R} \$ 17.218 .456,00$ & Lindbergh Farias & $\mathrm{R} \$ 17.965 .000,00$ \\
Renan Calheiros & $\mathrm{R} \$ 6.296 .796,00$ & Itamar Franco & $\mathrm{R} \$ 8.771 .137,00$ & Marta Suplicy & $\mathrm{R} \$ 8.635 .000,00$ \\
Simone Tebet & $\mathrm{R} \$ 6.020 .000,00$ & Aécio Neves & $\mathrm{R} \$ 5.363 .215,00$ & Maria de Fatima & $\mathrm{R} \$ 3.610 .000,00$ \\
Eunicio Lopes & $\mathrm{R} \$ 5.600 .000,00$ & Cassio Cunha Lima & $\mathrm{R} \$ 3.900 .000,00$ & José Pimentel & $\mathrm{R} \$ 3.610 .000,00$ \\
Edison Lobão & $\mathrm{R} \$ 5.600 .000,00$ & Flexa Ribeiro & $\mathrm{R} \$ 3.000 .000,00$ & Gleise Hoffman & $\mathrm{R} \$ 3.420 .000,00$ \\
José Maranhão & $\mathrm{R} \$ 3.400 .000,00$ & Lucia Costa & $\mathrm{R} \$ 1.800 .000,00$ & Paulo Rocha & $\mathrm{R} \$ 2.850 .000,00$ \\
Acir Gurcaz & $\mathrm{R} \$ 1.866 .656,00$ & Paulo Bauer & $\mathrm{R} \$ 1.360 .000,00$ & PC DO B & $\mathrm{R} \$ 2.195 .538,00$ \\
Carlos Braga & $\mathrm{R} \$ 1.780 .000,00$ & Alvaro Dias & $\mathrm{R} \$ 1.200 .000,00$ & PCB & $\mathrm{R} \$ 2.100 .000,00$ \\
DEM & $\mathrm{R} \$ 1.600 .000,00$ & PDT & $\mathrm{R} \$ 816.000,00$ & José Wellignton & $\mathrm{R} \$ 1.615 .000,00$ \\
Jader Barbalho & $\mathrm{R} \$ 1.200 .000,00$ & PSD & $\mathrm{R} \$ 576.000,00$ & PR & $\mathrm{R} \$ 1.542 .200,00$ \\
\hline
\end{tabular}

A análise descrita até aqui permitiu responder a duas das perguntas formuladas neste estudo: (i) quais são as empresas que mais doam e como distribuem suas doações e (ii) qual é o papel dos partidos e das suas lideranças na distribuição das doações. A terceira pergunta - qual o grau de poder que cada empresa possui sobre cada partido e senador - 
será respondida avaliando cada aresta ligada aos 81 senadores, formando uma matriz que pode ser considerada um mapa de intenções do Senado Brasileiro. Por limitações de espaço deste artigo apresentaremos essa matriz apenas para alguns relacionamentos entre empresas-partidos e empresas-senadores.

Na Tabela 4 podemos ver que a empresa JBS S.A. possui poder (métrica positiva) sobre quase todos os partidos, com exceção do PSDB e PMDB, com uma diferença significativa em relação às outras duas empresas apresentadas.

Tabela 4. Empresas e seu poder em relação aos maiores partidos do Senado

\begin{tabular}{lrrrrrrrrrr}
\hline Empresa & PSDB & PMDB & PT & PSD & PSB & PP & PR & DEM & PTB & PDT \\
\hline JBS S.A. & $-0,032$ & $-0,082$ & 0,001 & 0,131 & 0,020 & 0,188 & 0,142 & 0,001 & 0,062 & 0,146 \\
Const. Andrade Gutierrez S.A. & $-0,354$ & $-0,401$ & $-0,069$ & $-0,003$ & $-0,001$ & $-0,001$ & 0,001 & 0,018 & 0,034 & sem aresta \\
Amil Assis. Méd. Internac. S.A. & $-0,180$ & $-0,399$ & $-0,053$ & $-0,021$ & $-0,014$ & $-0,024$ & $-0,045$ & $-0,015$ & $-0,006$ & sem aresta \\
\hline
\end{tabular}

As Tabelas 5, 6 e 7 mostram o perfil de alguns senadores em relação aos seus maiores doadores. Analisando os dados é possível perceber que Renan Calheiros é fortemente vinculado ao seu partido, que exerce alto poder sobre o senador, praticamente sem nenhuma disputa com empresas externas. Já no caso de Lindbergh Farias, apesar do partido ser a força mais potente, vemos empresas atuando com poder positivo sobre o senador. Em contraste, o senador Blairo Maggi não possui nenhum ator que possua ascendência sobre ele nos termos da nossa análise. Detendo um volume enorme de recursos, o senador é empoderado em relação a todos os atores, inclusive o seu partido. Isto pode ser visto pelos valores negativos da métrica, que indicam que o lado que recebe o recurso é mais forte que o lado que distribui.

Tabela 5. Perfil de poder de Renan Calheiros e seus maiores doadores

\begin{tabular}{lr}
\hline Doador & poder \\
\hline PMDB & 0,879 \\
Franere Com. Const. e Imobiliária Ltda. & $-0,96$ \\
SOTAN - Sociedade de Táxi Aéreo do Nordeste Ltda. & $-0,99$ \\
\hline
\end{tabular}

Tabela 6. Perfil de poder de Lindbergh Farias e seus maiores doadores

\begin{tabular}{lr}
\hline Doador & poder \\
\hline PT & 0,455 \\
Camargo Correa S.A. & 0,025 \\
UTC Engenharia S.A. & 0,024 \\
\hline
\end{tabular}

Tabela 7. Perfil de poder de Blairo Maggi e seus maiores doadores

\begin{tabular}{lc}
\hline Doador & poder \\
\hline Recursos próprios & $-0,827$ \\
Construtora Sanches Tripoloni Ltda. & $-0,886$ \\
Amaggi Exportação e Importação Ltda. & $-0,908$ \\
\hline
\end{tabular}


É possível avaliar ainda o poder que os partidos exercem uns sobre os outros, uma vez que identificamos que grandes partidos agem neste sentido. A Tabela 8 mostra como os principais partidos atuam sobre os demais. A disputa de poder é bastante evidente pelos valores praticamente em equilíbrio da métrica, com alguns destaques: PSDB, PMDB e PT distribuem recursos principalmente para partidos cujo alinhamento não é claro, como PR, PTB e PDT. Partidos com uma posição mais alinhada com determinado ator não recebem recursos de seus adversários. É o caso do DEM e Solidariedade, que não recebem recursos do PT, assim como o PC do B, que não recebe recursos do PSDB e PMDB.

Tabela 8. Partidos e seu poder em relação a outros partidos

\begin{tabular}{lrrrrrrrr}
\hline Partido & PP & PR & DEM & SD & PROS & PTB & PDT & PC do B \\
\hline PSDB & $-0,0023$ & $-0,0062$ & $-0,0014$ & $-0,0002$ & $-0,0002$ & $-0,0013$ & $-0,0024$ & sem aresta \\
PMDB & sem aresta & $-0,0033$ & $-0,0076$ & $-0,0014$ & $-0,0047$ & $-0,0005$ & 0,0024 & sem aresta \\
PT & sem aresta & $-0,0164$ & sem aresta & sem aresta & sem aresta & $-0,0002$ & 0,0013 & 0,0292 \\
PSD & sem aresta & sem aresta & sem aresta & sem aresta & sem aresta & $-0,1634$ & sem aresta & $-0,0985$ \\
PSB & $-0,0232$ & sem aresta & sem aresta & $-0,0214$ & $-0,0013$ & sem aresta & sem aresta & $-0,0369$ \\
\hline
\end{tabular}

\section{Conclusões e Trabalhos Futuros}

Para avaliar as métricas propostas este estudo criou e analisou a rede de poder do Senado Federal em relação às empresas que doaram recursos para as campanhas de 2010 e 2014. Buscou-se avaliar quanto poder as empresas têm sobre partidos e senadores, através da visão de reciprocidade desta relação. A análise revelou alguns dados interessantes: nem sempre o maios valor doado corresponde a um maior poder sobre o partido ou senador; os grandes partidos têm muito poder sobre os partidos menores, intermediando a relação entre os mesmos e as empresas; alguns senadores se destacam de seus partidos; grandes empresas doadoras tendem a concentrar as doações em partidos e não em comitês individuais de senadores.

É importante frisar que a métrica poder (power) é um atributo da relação entre os nós e não dos nós em si. Uma limitação deste trabalho é que o cálculo não leva em conta o número de parceiros, apenas o volume de recursos. Um refinamento na fórmula de modo a considerar o número de opções de troca seria consistente com a base teórica utilizada. Outro ponto é que sua verificação foi feita em uma rede social específica e uma avaliação mais genérica certamente se faz necessária. A verificação da relação entre a métrica power e as métricas de hub e authority dos nós ligados pode trazer vínculos entre a micro-estrutura da rede e a relação de poder, bem como a revisão de outras métricas frequentemente usadas para análise de influência.

Do ponto de vista dos dados eleitorais, esta análise pode ser expandida para os políticos em todas as esferas, incluindo as eleições municipais. Um exercício interessante pode ser verificar hipoteticamente como a exclusão de um nó importante afetaria as relações de poder da rede. Por fim, um importante passo é a criação de um método simples e público de visualização desta rede de poder de maneira que o cidadão esteja ciente dos interesses que motivam cada um de seus representantes.

\section{Referências}

Anttiroiko, A. (2015). Castells' network concept and its connections to social, economic and political network analyses. Journal of Social Structure, 16(11):1-18. 
Boas, T. C., Hidalgo, F. D., and Richardson, N. P. (2014). The spoils of victory: campaign donations and government contracts in brazil. The Journal of Politics, 76(02):415-429.

Castells, M. (1996a). The network society. Backwell, Oxford.

Castells, M. (1996b). The Rise of the Network Society. The information age: Economy, society, and culture. Backwell, Oxford.

Castells, M. (2011). Network theory- a network theory of power. International Journal of Communication, 5:15.

Easley, D. and Kleinberg, J. (2010). Networks, crowds, and markets: Reasoning about a highly connected world. Cambridge University Press, Cambridge.

Emerson, R. M. (1976). Social exchange theory. Annual review of sociology, 2(1):335362.

French, J. R., Raven, B., and Cartwright, D. (1959). The bases of social power. In Studies in social power, volume 7. Institute for Social Research, The University of Michigan.

Hindess, B. (1996). Discourses of power from Hobbes to Foucault. Backwell, Oxford.

MacLuhan, M., Fiore, Q., and Agel, J. (1967). The medium is the massage. Random House, New York.

Mancuso, W. P., Figueiredo Filho, D. B., Speck, B. W., Silva, L. E. O., and Rocha, E. C. d. (2016). Corporate dependence in brazil's 2010 elections for federal deputy. Brazilian Political Science Review, 10(3).

Marx, K. and Engels, F. (1968). Karl Marx and Frederick Engels: selected works in one volume, volume 184. International Publishers, New York.

Tribunal Superior Eleitoral (2016). Repositório de dados eleitorais. Disponível em http://divulgacandcontas.tse.jus.br. Acessado em 01 novembro de 2016.

Willer, D. (1999). Network exchange theory. Greenwood Publishing Group. 